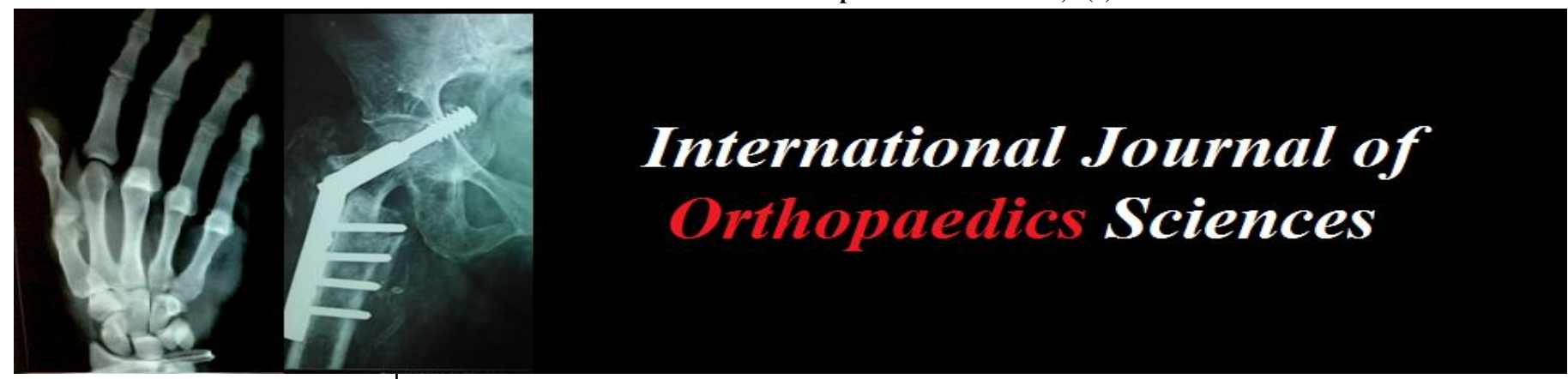

E-ISSN: 2395-1958

P-ISSN: 2706-6630

IJOS 2020; 6(2): 155-160

(C) $2020 \mathrm{IJOS}$

www.orthopaper.com

Received: 04-01-2020

Accepted: 08-02-2020

Dr. Prakashappa TH

Professor and Head of

Department, Department of Orthopaedics, Sanjay Gandhi

Institute of Trauma and

Orthopaedics, Bangalore,

Karnataka, India

Dr. Avinash P

Professor and Unit Head,

Department of Orthopaedics,

Sanjay Gandhi Institute of

Trauma and Orthopaedics,

Bangalore, Karnataka, India

Dr. Balaram Patil M

Post Graduate, Deparment of Orthopaedics, Sanjay Gandhi Institute of Trauma and Orthopaedics, Bangalore, Karnataka, India
Corresponding Author: Dr. Avinash P

Professor and Unit Head, Department of Orthopaedics, Sanjay Gandhi Institute of Trauma and Orthopaedics, Bangalore, Karnataka, India

\section{Functional outcome of distal humerus fracture in adults treated with Bicolumnar plating: A prospective study}

\author{
Dr. Prakashappa TH, Dr. Avinash P and Dr. Balaram Patil M
}

DOI: https://doi.org/10.22271/ortho.2020.v6.i2c.2030

\section{Abstract}

Background: Distal humerus fractures in adults are very difficult and challenging to treat due to intra articular and inter condylar involvement. It accounts for $2 \%$ to $6 \%$ of all fractures and one third of humerus fractures. The aim of this study was to evaluate the functional outcome of distal humerus fractures treated by bi columnar plating.

Materials and Methods: Our study consists of 30 post traumatic distal humerus fracture AO type C3 operated between December 2017 and December 2018. The functional outcome was assessed using The Mayo Elbow Performance Score. Patients were followed up at 6 weeks, 3months, 6months and 1 year post operatively.

Results: There were 21(70.0\%) male patients, and 9(30\%) female patients. Average age of the patients was 39.68 years with age ranging from 18 years to 80 years. $11(36.7 \%)$ cases were right humerus and $19(63.3 \%)$ were left sided humerus involved. All cases were operated through trans olecranon approach and chevron osteotomy technique, fracture stabilized with bi columnar locking compression plates. At the final follow up most of the patients had excellent postoperative elbow flexion with average arc of elbow flexion of 117.33 degrees, average extension deficit of 8.67 degrees, average pronation of 70.83 degrees, average supination of 70.83 degrees.

Conclusions: Bi columnar locking compression plates offer a good fixation for the distal humerus fractures with particular advantage in intra-articular type C3. The device helps in early mobilisation even in fractures with comminution due to its enhanced stability.

Keywords: Distal humerus fracture, Trans Olecranon, Chevron Osteotomy, Bi columnar locking compression plates, MEP Score.

\section{Introduction}

Distal humerus fractures in adults are very difficult and challenging to treat due to intra articular and inter - condylar involvement, high comminution, reconstruction difficulty of the articular surfaces, maintaining stability and morbidity, if not constructed anatomically. It accounts for $2 \%$ to $6 \%$ of all fractures and one third of humerus fractures ${ }^{[1]}$. Robinson reported that the incidence of distal humerus factures in adults was 5.7/100000 per year in a 10 year follow up study ${ }^{[2]}$. In a study conducted by Palvanen' elderly individuals aged (> 60 years) were predicted to have a three-fold rise in incidence by 2030 . Hence its important to understand these fractures and their treatment protocol ${ }^{[3]}$. Non-surgical treatment can be justified in cases of hemiplegia sequelae involving the ipsilateral upper limb, advanced osteoporosis and fractures with extensive bone loss but to avoid fixation failure, stiffening is almost assured and arthrolysis will have to be performed later on. The main goal of surgical treatment is to obtain fixation that is stable enough to allow immediate post operative elbow mobilization to prevent it from stiffening, mal - union and non - union, hence open reduction and internal fixation allows early mobilization and good functional outcomes. Trans olecranon approach and Chevron osteotomy technique exposes the articular surface of the distal humerus more than other approaches ${ }^{[4-7]}$. Patients who underwent Open reduction and internal fixation through an olecranon osteotomy showed good range of movement and good to excellent results than those who under went open reduction and internal fixation through other approaches ${ }^{[8,10]}$. 
Locking compression plates are more beneficial in distal humerus fractures than dynamic compression plates and $\mathrm{k}$ wires. Single columnar plating, Recon plating and conventional plates have more fixation failures. Complete fixation with two pre contoured anatomical locking compression plates in 90 degree is the most suitable technique for distal humerus fractures [11]. Patients treated with bi columnar locking compression plates show a greater functional range of motion, better restoration of articular congruity, secure bony fixation, better bone healing, and early rehabilitation ${ }^{[12]}$.

Materials and Methods: This was a prospective study consists of distal humerus fractures (AO type 13C) which presented to Sanjay Gandhi Institute Of Trauma and Orthopaedics, Bangalore between the period of December
2017 and December 2018, were included, after obtaining the permission from the Institutional Ethics Committee.

Inclusion criteria: Age more than 18 years belonging to both sexes with distal humerus fractures [AO type 31C], Closed and Early Gustilo-Anderson type 1 and 2 open fractures, Subjects with bilateral fractures were included.

Exclusion criteria: Age less than 18 years, Co-existing ipsilateral limb fractures, Gustilo-Anderson type 3 fractures, Closed fractures with neuro-vascular injuries, Pathological fractures, Revision surgeries or operated elsewhere, Upper limb congenital deformity, Established inflammatory or degenerative arthritis of the elbow and patients who refused to give informed written consent were excluded.

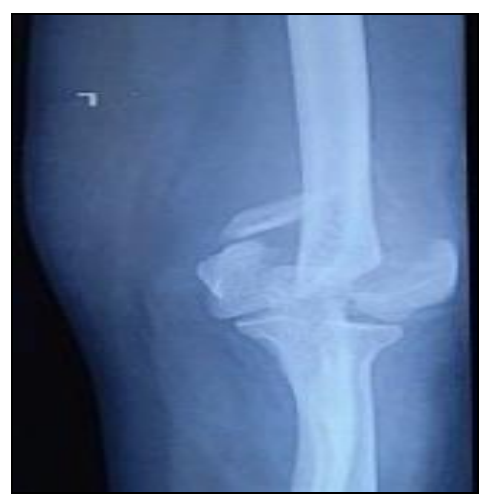

Fig 1: Pre op AP radiograph

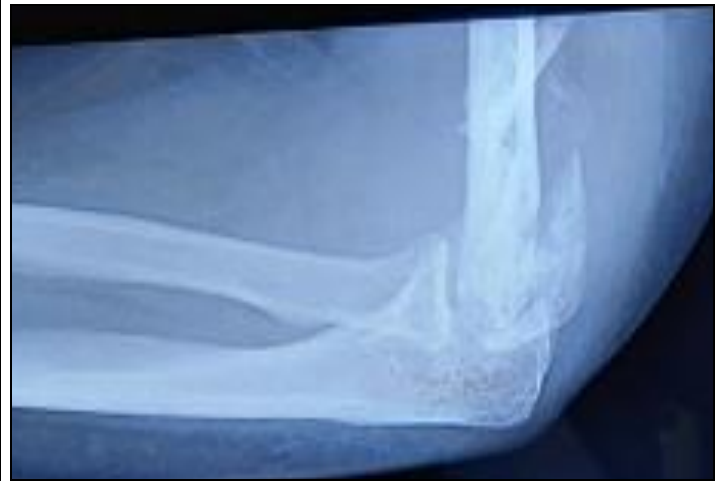

Fig 2: Pre op Lateral radiographs
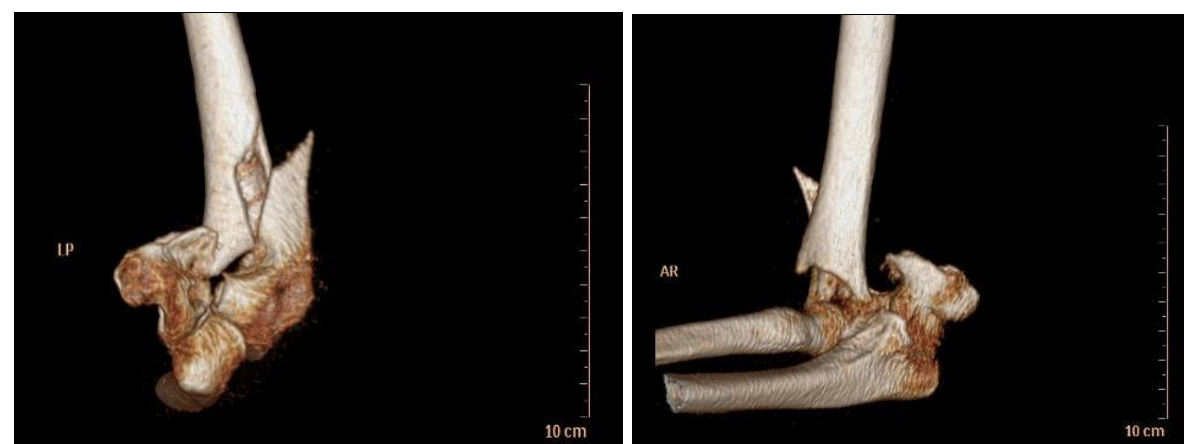

Fig 3: Pre op CT scan with 3D reconstruction.

Surgical Technique: All cases were operated by a senior trauma surgeon. Radiographs and CT images were reviewed for selection of plate size, plate position and for inter fragmentary screws. Under all aseptic medications and under general anaesthesia or regional Brachial plexus block, patient was positioned in lateral position with arm on a side bar and a high pneumatic tourniquet was used. Entire upper limb was painted and draped with a strict sterile technique, Posterior, midline longitudinal incision was used. Ulnar nerve was identified, retracted in all cases, Chevron olecranon osteotomy was done to expose the articular surface (Fig 1). Articular surface was visualized fully and morphology of the fracture was re-assessed. Inter - condyles were fixed with cannulated cancellous screws. Fracture fixed with bicolumnar, orthogonal plating technique using AO Synthes $®$ fixed angle locking compression plates (Fig 2). Osteotomy repaired by tension band wiring.

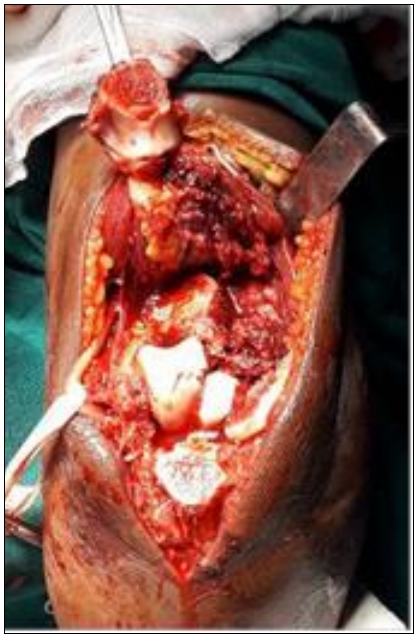

Fig 4: Chevron olecranon osteotomy 


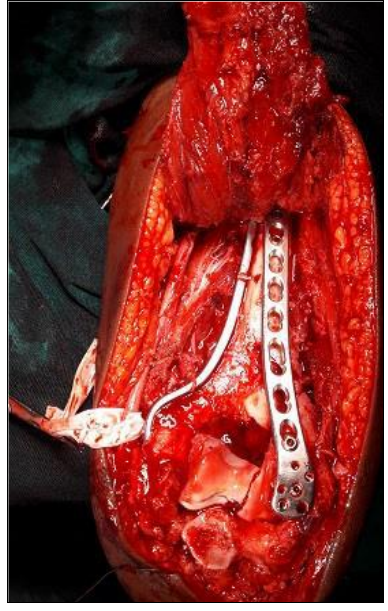

Fig 5: Bi columnar plating
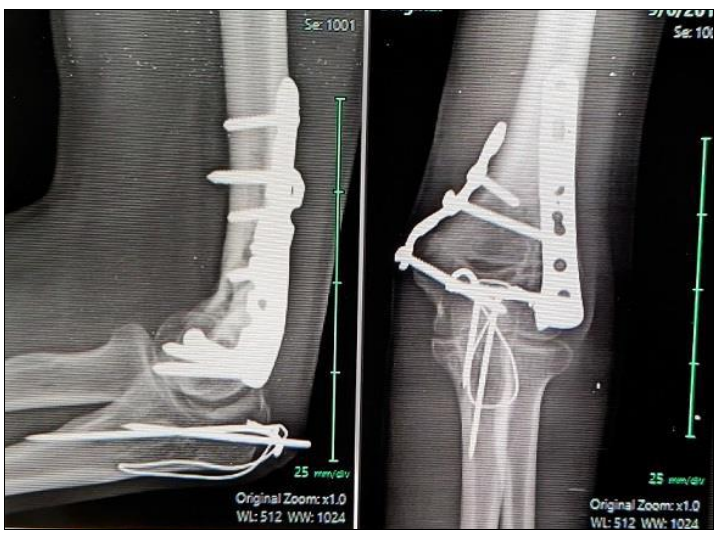

Fig 6: 6months follow up

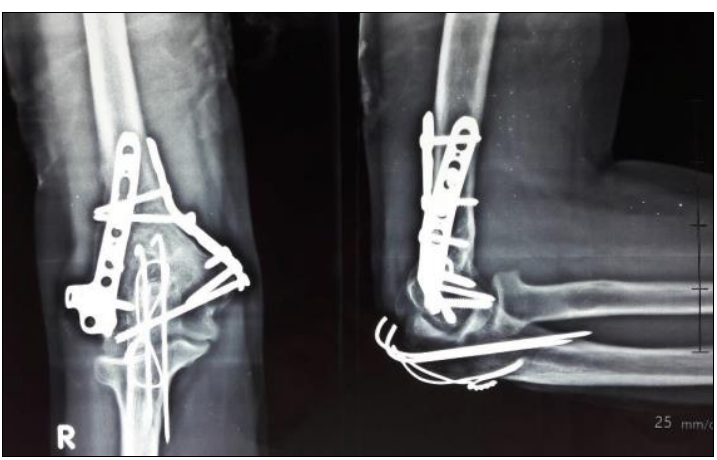

Fig 7: 1 year follow up

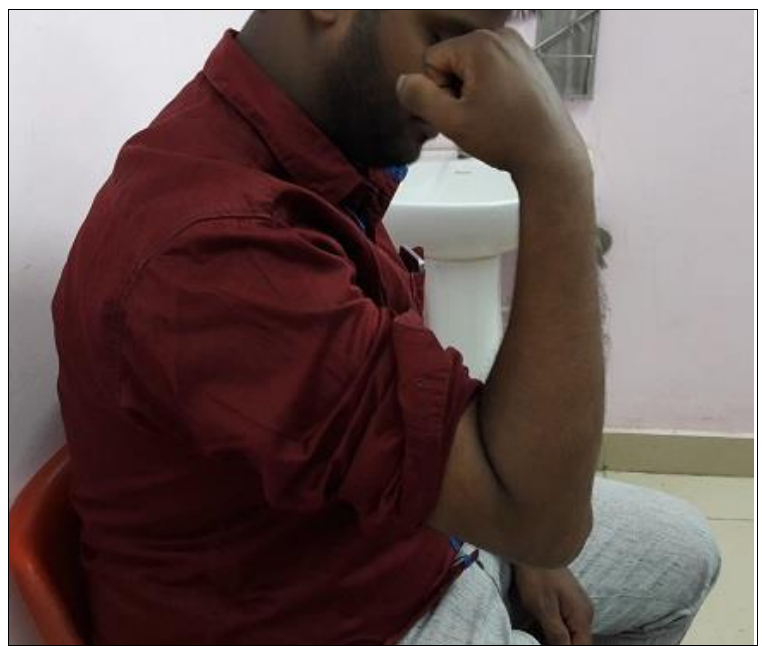

Fig 8: Flexion at 1 year

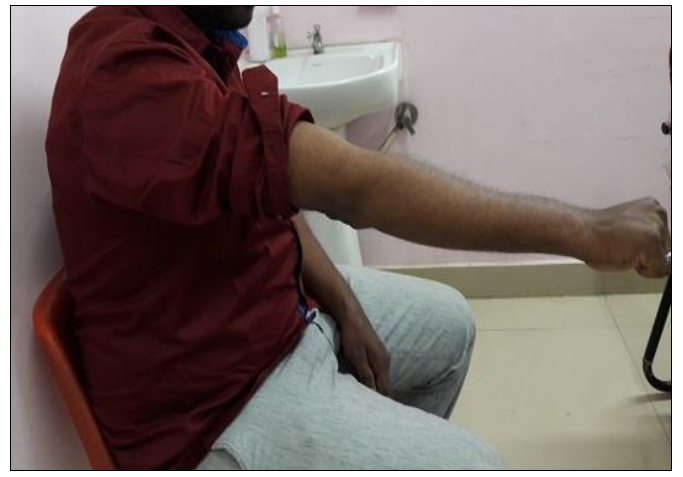

Fig 9: Extension at 1 year

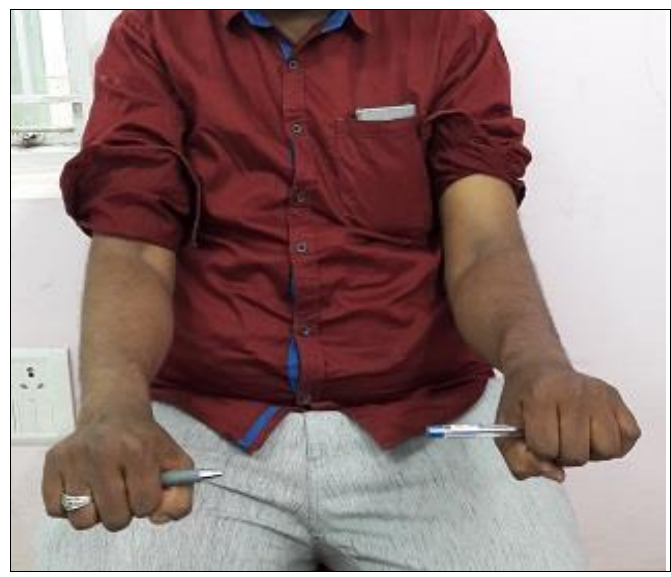

Fig 10: Pronation at 1 year

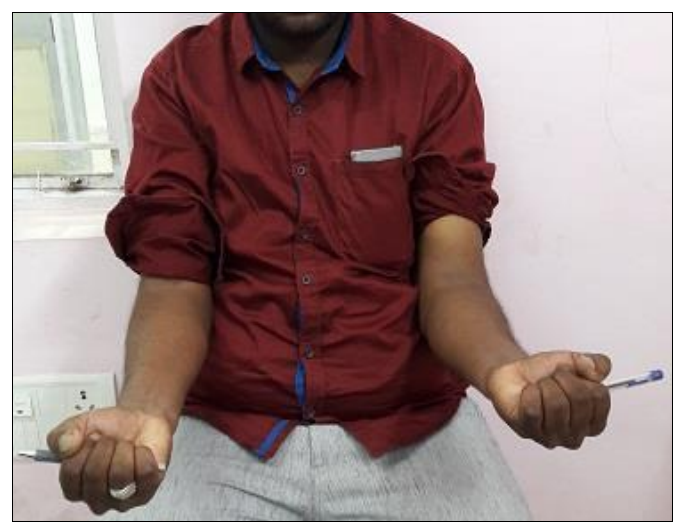

Fig 11: Supination at 1 year

Post operative management: Post operatively the patient was immobilized with above elbow slab with elbow in $90^{\circ}$ flexion and forearm in supine position for a period of two weeks. Third generation cephalosporins was administered parenteral for 48 hours post-operatively till the drain tubes were removed. Then oral antibiotic was started and continued till the suture removal. Oral indomethacin $(75 \mathrm{mg} /$ day) was started on post-op day one and continued for three weeks. Active assisted mobilization was started after suture removal under the supervision of a senior physiotherapist. Patients were evaluated at 6 weeks, 3 months, 6 months and 1 year from December 2017. End point of follow up was 1 year. In the follow up wound related complications (Infection), range of movements at elbow joint and distal radio ulnar joint, return to professional / routine activities, ulnar neuropathy, fracture union, implant failure and heterotopic ossification were assessed. Functional outcome was assessed by Mayo elbow performance score. 
Results: The mean age was 39.68, with the youngest being 21 years and oldest is 80 years(Table 1). 21 males and 9 females included in this study (Table 2). In 19 patients left side was involved and in 11 patients right side involved (Table 3). 15 cases were due to Road traffic accidents and 15 cases due to self fall (Table 4). 29 patients had a closed type and only one patient had an open type II fracture. Out of 30 patients only one patient did not return to his prior activities. 3 patients had superficial skin infection and they underwent early removal of protruding ' $\mathrm{K}$ ' wires from TBW site after union at osteotomy site and parenteral antibiotics were started according to culture and sensitivity following which the infection subsided. Two patients had post operative ulnar neuritis in their early follow up (less than 3 months). The main complaint was paresthesisa in the ulnar nerve distribution, they had no motor deficits and are treated symptomatically. However at the final follow up none of them had ulnar neuritis.

Table 1: age distribution

\begin{tabular}{|c|c|c|}
\hline Age (in years) & No of patients & Percentage \\
\hline $18-38$ & 18 & $60.00 \%$ \\
\hline $39-58$ & 6 & $20.00 \%$ \\
\hline $59-78$ & 4 & $13.33 \%$ \\
\hline$>78$ & 2 & $6.67 \%$ \\
\hline Total & 30 & $100 \%$ \\
\hline
\end{tabular}

Table 2: Sex distribution

\begin{tabular}{|c|c|c|}
\hline Gender & No of cases & Percentage \\
\hline Male & 21 & $70.0 \%$ \\
\hline Female & 9 & $30.0 \%$ \\
\hline Total & 30 & $100 \%$ \\
\hline
\end{tabular}

Table 3: Side involvement.

\begin{tabular}{|c|c|c|}
\hline Side & No of cases & Percentage \\
\hline Left & 19 & $63.3 \%$ \\
\hline Right & 11 & $36.7 \%$ \\
\hline Total & 30 & $100 \%$ \\
\hline
\end{tabular}

Table 4: Mode of injury.

\begin{tabular}{|c|c|c|}
\hline Mode of Injury & Number of cases & Percentage \\
\hline RTA & 15 & $50 \%$ \\
\hline Self-fall & 15 & $50 \%$ \\
\hline Total & 30 & $100 \%$ \\
\hline
\end{tabular}

MEPS (Table 5) was excellent in 21 patients, good in 5 patients, fair in 3 patients and poor in 1 patient. The patient with poor score had severe stiffness and experienced difficulty in performing daily routine activities.

Table 5: Mayo elbow performance score.

\begin{tabular}{|c|c|c|c|c|}
\hline MEPS & 6 Weeks & 3 Months & 6 Months & 1 Year \\
\hline Excellent & $0(0)$ & $3(10.0 \%)$ & $11(36.7 \%)$ & $21(70.0 \%)$ \\
\hline Good & $7(23.3 \%)$ & $18(60.0 \%)$ & $15(50.0 \%)$ & $5(16.7 \%)$ \\
\hline Fair & $16(53.3 \%)$ & $7(23.3 \%)$ & $2(6.7 \%)$ & $3(10.0 \%)$ \\
\hline Poor & $7(23.3 \%)$ & $2(6.7 \%)$ & $2(6.7 \%)$ & $1(3.3 \%)$ \\
\hline
\end{tabular}

Paired ' $t$ ' test was used to analyse the comparision of MEPS between different time intervals. A ' $p$ ' value of $<0.001$ was obtained signifying that the comparision was statistically highly significant in every follow up (Table 6).
Table 6: Comparison of MEPS between different time intervals.

\begin{tabular}{|c|c|c|c|c|c|}
\hline \multirow{2}{*}{ MEPS } & \multirow{2}{*}{ N } & \multirow{2}{*}{ Mean } & $\begin{array}{c}\text { Std. } \\
\text { Deviation }\end{array}$ & \multicolumn{2}{|c|}{ Repeated Measures ANOVA } \\
\cline { 1 - 1 } 6 Weeks & 30 & 63.00 & 10.22 & & p-value \\
\hline 3 Months & 30 & 76.83 & 11.02 & \multirow{2}{*}{176.18} & \multirow{2}{*}{$<0.001^{*}$} \\
\hline 6 Months & 30 & 84.33 & 11.28 & & \\
\hline 1 Year & 30 & 92.00 & 13.37 & & \\
\hline
\end{tabular}

Discussion: We studied 30 cases of distal humerus fractures AO type 13-C over a period of 1 year, all were intra articular fractures and we evaluated their functional outcome. Palvanen and Robinson observed in their respective studies, a bimodal age distribution ${ }^{[2,3]} .18$ patients aged $18-38$ years and rest of the 11 patients more than 40 years. In our study 15 patients had Road traffic accident and 15 had self fall, 11 had a trivial fall from standing height, indicating that osteoporosis may be a pre - disposing factor. Osteoporosis is the major risk factor leading to these type of fractures in the elderly ${ }^{[3]}$. Even though the elbow joint is superficial posteriorly, open fractures are not common, if present with open fracture it will be due to high velocity injury. Min et al. stated in their study that open distal humerus fractures are about $2.7 \%$ of all the open long bone fractures, $85.7 \%$ of these fractures are $\mathrm{AO}$ type C $3{ }^{[22]}$. A single trans - olecranon approach and single technique was applied for all the fractures, chevron osteotomy was done in all the cases for good articular visualization and then the fracture was fixed with $3.5 \mathrm{~mm}$ bi columnar locking compression plates. Wilkinson and Stanley recommended in their studies that olecranon osteotomy exposes the articular surface fully than other approaches. ${ }^{5}$ Jupiter stated in his study that chevron olecranon osteotomy is the workhorse for exposing the articular surface. ${ }^{9}$ Gofton and Kundel used Chevron olecranon osteotomy and obtained good to excellent outcomes $[10,23]$. We used trans olecranon approach with Chevron osteotomy which gave very good visualization of the articular surface and it gave sufficient working space to attain anatomic reduction and excellent result with respect to the ability to reconstruct the articular surface.

Open reduction and internal fixation with bi - columnar locking compression plates is the gold standard treatment for distal humerus AO type $3 \mathrm{C}$ fractures. However, the position of the two plates with respect to each other has been a matter of discussion. The traditional concept was to place the plates in an orthogonal fashion (two perpendicular planes) and this has been challenged by the parallel plating technique. There are three studies in the literature which have compared the two techniques. While Jacobson and his colleague concluded that orthogonal plating provided better stability to the construct, Schemitsch and Self concluded the contrary, stating parallel plating technique was better ${ }^{[24-26]}$. Gofton, Kundel and Aslam all obtained excellent to good functional outcome with orthogonal plating technique ${ }^{[10,24,27]}$. Further, Korner compared fixation using locking compression plates (LCP) with conventional reconstruction plates and opined that LCP provides better stability, especially in areas where screw purchase is poor and also provides an increased resistance to bending, torsion and axial compression loading as compared to conventional plates ${ }^{[1]}$. The locking capability is important for a fixed angle construct in osteopenic bone or multifragmentary fractures where screw purchase is compromised. 
We followed orthogonal plating using $3.5 \mathrm{~mm}$ fixed angled AO Synthes ${ }^{\circledR}$ anatomic locking compression plates and obtained excellent to good outcome. This technique offered adequate stability of the construct to permit early mobilization. Gofton reported about loss of initial reduction and $40 \%$ of his patients required an additional plate to achieve stability. However, we did not come across such an event ${ }^{[10]}$. In our study, a mean arc of flexion of $117.33^{\circ}$ with a mean extension deficit of $8.67^{0}$ was observed. This is comparable to that obtained by Gofton, Kundel and Aslam in their study on AO type $3 \mathrm{C}$ fractures of the distal humerus. ${ }^{10,24,28} 21$ patients had excellent score, 5 had good score, 3 fair and 1 poor on MEPS. 29 patients had returned to their regular routine activities at the time of final follow up. This is also in comparison to those mentioned in the literature (Table 7).

Table 7: Comparision of our study with those in literature

\begin{tabular}{|c|c|c|c|c|}
\hline Reference & $\begin{array}{c}\text { No. of Patients Followed } \\
\text { up }\end{array}$ & $\begin{array}{c}\text { No.of Excellent to good } \\
\text { results }\end{array}$ & $\begin{array}{c}\text { Mean arc Of Flexion } \\
\text { in deg }\end{array}$ & $\begin{array}{c}\text { No. of } \\
\text { Complications / type }\end{array}$ \\
\hline Gofton & 23 & 21 & 122 & $7 \mathrm{HO}, 2$ infection, 3 non union \\
\hline Aslam & 20 & 14 & 122 & 2 HO, 2 infection, 1 ulnar neuritis \\
\hline Kundel & 77 & 40 & 104 & 29 HO, 8 infection, 21 Ulnar neuritis \\
\hline This study & 30 & 26 & 117 & 3 Infection, 2 ulnar neuritis \\
\hline
\end{tabular}

Radio logically, all the fractures showed union at final followup. Helfet reported non-union rate of distal humerus fractures treated by ORIF at $2-10 \%{ }^{[6]}$. We did not come across implant loosening or implant breakage leading to loss of fixation. In literature we came across authors observing non - union, implant failure and Heterotopic Ossification on post-operative radiographs. The common complications seen following surgical management of distal humerus AO type $3 \mathrm{C}$ fractures as noted by Jupiter in a review article include elbow stiffness, infection, non-union, ulnar neuropathy and heterotopic ossification (HO) ${ }^{[9]}$. Although almost all patients in our study had certain degree of elbow of stiffness, 29 of them had a functional range of movement. This is comparable to studies in the literature. One patient had severe stiffness with only $10^{0}$ movement in the flexion-extension arc with a fixed-flexion deformity of $40^{\circ}$. Helfet observed non-union in $2-10 \%$ of patients treated by ORIF, and it is common in patients with severe comminution, bone loss and inadequate fixation ${ }^{[6]}$. Jupiter and Green ${ }^{[2]}$ reviewed the literature and reported that post-operative infection in these fractures range from 0 to $9 \%$ $[9,28]$. We had 3 patients presenting with postoperative wound infection, which was superficial skin infection, K-wires and SS wires were removed and a thorough wound wash was given. With antibiotics sensitive to the aerobic culture report, the infection subsided in due course and required no further intervention. None of the other patients had any wound related complications. Transient ulnar neuritis is a common post-operative complication described in the literature. Kundel, Yilmaz and Aslam all have reported transient ulnar neuritis in the post-operative period. There has been a debate as to whether routine anterior transposition of the ulnar nerve during the exposure has any role in preventing postoperative ulnar neuritis ${ }^{[23,27,29]}$. Gofton, Eralp and Wang demonstrated less than $1 \%$ incidence of ulnar neuritis following routine transposition, as opposed to Helfet $^{6}$ who showed $7 \%$ incidence on not performing the transposition ${ }^{[6,10,30,31]}$. This opinion was, however, challenged by Chen and Vazquez who advised against transposition [32, 33]. Chen, in his study, concluded that routine transposition is associated with higher incidence of post-operative ulnar neuritis ${ }^{[32]}$. There is, hence, a conflict as to perform the transposition or not. In all the cases, we identified the ulnar nerve by dissection along the muscle fibre and protected it during the surgery. Care was taken to avoid excessive devascularisation and forceful retraction. However, we did not perform anterior transposition in any of the cases. We had two patients presenting with symptoms of ulnar neuritis, mainly paraesthesia. All of them developed the symptoms within 6 weeks postoperatively. None of them had motor deficits. All were managed symptomatically. At final follow-up, none of them had any residual symptoms.

Conclusion: In conclusion, this study shows that distal humerus fractures operated with bicolumnar locking compression plates using trans olecranon approach by chevron osteotomy has good functional outcome with particular advantage in the intra-Articular type C3 fractures, which allows early mobilisation of the patients even in fractures with comminution due to the enhanced stability of the construct.

\section{References}

1. Anglen J. Distal humerus fractures [review]. J Am Acad Orthop Surg. 2005; 13:291-7.

2. Robinson CM, Hill RM, Jacobs N. Adult distal humeral metaphyseal fractures: epidemiology and results of treatment. J Orthop Trauma. 2003; 17:38-47.

3. Palvanen M, Kannus P, Parkkari J. The injury mechanisms of osteoporotic upper extremity fractures among older adults: a controlled study of 287 consecutive patients and their 108 controls. Osteoporos Int. 2000; 11:822-31.

4. O'Driscoll SW. Supracondylar fractures of the elbow: open reduction, internal fixation [review]. Hand Clin. 2004; 20:465-74.

5. Wilkinson JM, Stanley D. Posterior surgical approaches to the elbow: a comparative anatomic study. J Shoulder Elbow Surg. 2001; 10:380-2.

6. Helfet DL, Schmeling GJ. Bicondylar intra-articular fractures of the distal humerus in adults [review]. Clin Orthop Relat Res. 1993; 292:26-36.

7. Pollock JW, Faber KJ, Athwal GS. Distal humerus fractures. Orthop Clin N Am. 2008; 39(2):187-200.

8. Pajarinen J, Bjorkenheim JM. Operative treatment of type $\mathrm{C}$ intercondylar fractures of the distal humerus: results after a mean follow-up of 2 years in a series of 18 patients. J Shoulder Elbow Surg. 2002; 11:48-52.

9. Jupiter JB, Mehne DK. Fractures of the distal humerus [review]. Orthopaedics. 1992; 15:825-33.

10. Gofton WT McDermid JC, Patterson SD, Faber KJ, King GJW. Functional outcome of AO type C distal humerus fractures. J Hand Surg. Am. 2003; 28(2):294-308.

11. Korner J, Diederichs G, Arzdorf $\mathrm{M}$ et al. A biomechanical evaluation of methods of distal humerus 
fracture fixation using locking compression plates versus conventional reconstruction plates. J Orthop Trauma. 2004; 18:286-93.

12. Ring D, Gulotta L, Jupiter JB. Unstable non unions of the distal part of the humerus. J Bone Joint Surgery Am. 2003; 85-A(6):1040-1046.

13. Yang KH, Park HW, Park SJ et al. Lateral J-plate fixation in comminuted intercondylar fracture of the humerus. Arch Orthop Trauma Surg. 2003; 123:234-8.

14. O'Driscoll SW, Sanchez-Sotelo J, Torchia ME. Management of the smashed distal humerus [review]. Orthop Clin North Am. 2002; 33:19-33.

15. Sanders RA, Raney EM, Pipkin S. Operative treatment of bicondylar intraarticular fractures of the distal humerus. Orthopaedics. 1992; 15:159-63.

16. McCarty LP, Ring D, Jupiter JB. Management of distal humerus fractures [review]. Am J Orthop. 2005; 34:4308 .

17. Gabel GT, Hanson G, Bennett JB. Intra-articular Fractures of Distal Humerus in the Adult. Clin Orthop. 1987; 216:99-108.

18. Ring D, Jupiter JB. Complex fractures of the distal humerus and their complications [review]. J Shoulder Elbow Surg. 1999; 8:85-97.

19. Holdsworth BJ, Mossad MM. Fractures of the adult distal humerus. Elbow function after internal fixation. J Bone Joint Surg. Br. 1990; 72:362-5.

20. O' Driscoll SW. Optimizing stability in distal humeral fracture fixation. J Shoulder Elbow Surg. 2005; 14:186S$194 \mathrm{~S}$.

21. Henley MB, Bone LB, Parker B. Operative management of intra-articular fractures of the distal humerus. J Orthop Trauma. 1987; 1:24-35.

22. Min W, Anwar A, Ding BC, Tejwani NC. Open distal humerus fractures: review of literature. Bulleting of the NYU Hospitals for joint diseases. 2010; 68(4): 257-61.

23. Kundel K, Braun W, Wieberneit J, Ruter A.Intraarticular distal humerus fractures. Factors affecting functional outc ome. Clin Orthop RelatRes 332:200208 Factors affecting functional outcome, 1996.

24. Jacobson SR, Glisson RR, Urbaniak JR. Compari-son of distal humerus fracture fixation: a biomechanical study. J South Orthop Assoc. 1997; 6:241-9.

25. Schemitsch EH, Tencer AF, Henley MB. Biomechanical evaluation of methods of internal fixation of the distal humerus. J Orthop Trauma. 1994; 8:468-75.

26. Self J, Viegas SF, Buford WL Jr, et al. A comparison of double-plate fixation methods for complex distal humerus fractures. J Shoulder Elbow Surg 1995; 4:10

27. Aslam N, Willet K. Functional outcome following internal fixation of intra-articular fractures of the distal humerus (AO type C). Acta Orthop Belg. 2008; 70(2):118-22.

28. Green DP, Wolfe S.W. 2011. Green's Operative Hand Surgery. Philadelphia: Saunders/Elsevier.

29. Yilmaz E, Bulut M. Outcomes of distal intra-articular humerus fractures treated by olecranon osteotomy. Dicle Med J. 36; 4: 241-247. Functional outcome. J Clin Orthop. 1996; 332:200-208.

30. Eralp L, Kocaoglu M, Sar C et al. Surgical treatment of distal intra-articular humeral fractures in adults. Int. Orthop. 2001; 25:46-50.

31. Wang KC, Shih HN, Hsu KY et al. Inter condylar fractures of the distal humerus: routine anterior subcutaneous transposition of the ulnar nerve in a posterior operative approach. J Trauma. 1994; 36:770-3.

32. Chen RC, Harris DJ, Leduc S, Borrelli JJ, Tornetta P, Ricci WM. Is ulnar nerve transposition beneficial during open reduction internal fixation of distal humerus fracture? J Orthop Trauma. 2010; 7:391-94.

33. Vizquez O, Rutgers M, Ring DC, Walsh M, Egol KA. Fate of the ulnar nerve after operative fixation of the distal humerus. J Orthop Trauma. 2010; 7:395-99. 